\title{
Clinical Evaluation in 80 Patients with Renovascular Hypertension
}

\author{
Keishi Abe, MD, Yoichi Otsuka, M D, Tetsuo Saito, M D, \\ Nobuo Irokawa, M D, Haruyuki Aoyagi, M D, Seiji Miyazaki, M D, \\ Takashi Kusaka, M D, Masahide Seino, M D, Minoru Yasujima, M D, \\ Satoru Chiba, M D, Yutaka SAKurai, M D, Keitaro Saito, M D \\ and Kaoru Yoshinaga, M D
}

The Second Department of Internal Medicine, Tohoku University School of Medicine

\begin{abstract}
Eighty cases of renovascular hypertension including 47 with unilateral main renal arterial stenosis (UMRAS), 16 bilateral main renal arterial stenoses (BMRAS), 15 renal arterial branch stenosis (RABS) and 2 unilateral main renal arterial stenoses with contralateral renal arterial branch stenoses were studied. Abnormal findings were observed in $84 \%$ of UMRAS, $73 \%$ of BMRAS and $74 \%$ of RABS by rapid sequence pyelographic study, and in $84 \%$ of UMRAS, $63 \%$ of BMRAS and $54 \%$ of RABS by radioisotope renographic study. The ischemic pattern was obtained in $69 \%$ of UMRAS, 50\% of BMRAS and $40 \%$ of RABS in split renal function test. High resting peripheral vein blood plasma renin activity values were obtained in $67 \%$ of UMRAS, but in only $33 \%$ of BMRAS and RABS. Hyperresponsiveness of renin secretion was found in $80 \%$ of BMRAS and $56 \%$ of RABS. A significant elevation of PRA in the stenotic kidney was found in $78 \%$ of UMRAS and $50 \%$ of unilateral RABS. Angiographic study revealed unilateral vascular lesion in 60 cases and bilateral in 19 cases. Development of collateral circulation was found in $73 \%$ of cases. Forty-nine cases underwent a surgical treatment and 40 cases of them were cured or improved. The most reliable diagnostic and prognostic criteria for surgical curability were to demonstrate collateral circulation and elevated peripheral vein PRA levels with increased renin secretion from the affected kidney. The postoperative course of blood pressure was examined with regard to the PRA in 18 nephrectomized and in 12 revascularized patients. The normalization of blood pressure was quicker in the latter than in the former, while the normalization of PRA was quicker in the former than in the latter.
\end{abstract}

Key Words: Renal arterial stenosis, Renal arterial branch stenosis, Plasma renin activity, Provocation test of renin.

Renovascular hypertension is one of the most common causes of correctable hypertension in which reduced renal arterial pressure is responsible for the

Received for publication December 12, 1975

Reprint requests to: Keishi Abe, The Second Department of Internal Medicine, Tohoku University School of Medicine, 1-1, Seiryomachi, Sendai 980, Japan. enhancement of renin secretion. ${ }^{1)}$ An increased release of renin is considered to be the cause of this type of hypertension. In chronic renovascular hypertension, however, it has not been fully confirmed that renin is involved in the maintenance of high blood pressure. Normal plasma renin activity was frequently found in renovascular hypertension, especially in 
the cases of bilateral renal arterial stenoses or branch arterial stenosis, but the hypertension can be relieved by surgical treatment. ${ }^{2,3)}$ On the other hand, there were several cases in whom the hypertension persisted after the reconstructive surgery or nephrectomy had been successfully performed. ${ }^{4)}$ In addition, renal arterial stenosis has been observed in normotensive patients. ${ }^{5)}$ It is therefore very important to establish the diagnosis preoperatively that the arterial lesion is a main cause of hypertension in the patients with renal arterial stenosis.

In the present report, 80 cases of renovascular hypertension treated for the past 13 years in our clinic were analysed retrospectively to evaluate the diagnosis, surgical curability and postoperative course of blood pressure with regard to plasma renin activity in nephrectomized or in revascularized cases.

\section{MATERIALS AND METHODS}

During the last 13 years, 80 cases of renovascular hypertension, including 47 of unilateral main renal arterial stenosis (UMRAS), 16 bilateral main renal arterial stenoses (BMRAS), 15 renal arterial branch stenosis (RABS) and 2 unilateral main renal arterial stenosis with contralateral renal arterial branch stenosis (UMCBS) have been studied in our clinic.

There were 43 men ranging in age from 10 to 66 (average 30.9 ) and 39 women from 12 to 60 (average 22.6). Blood pressure in men was from 162 to $246 \mathrm{mmHg}$ (average 206.4) in systole and from 60 to $156 \mathrm{mmHg}$ (average 110.3) in diastole. The patients having normal diastolic blood pressure belonged to atypical coarctation of the aorta or aortic arch type of aortitis syndrome with renal arterial stenosis. There were no significant differences between blood pressure levels of both sexes.

The diagnosis of renovascular hypertension was made by the following examinations; history, physical examinations, intravenous pyelography (rapid sequence pyelography), radioisotope renography, renoscintigraphy, split renal function test, abdominal aortography, and selective renal arteriography. All patients were hospitalized during this study period. They were maintained on regular diet containing approximately $250 \mathrm{mEq}$ of sodium or above daily, and antihypertensive therapy had been discontinued at least 2 weeks prior to the measurement of plasma renin activity.

Plasma renin activity (PRA) was measured partly by bioassay ${ }^{6,7)}$ and mostly by a method using angiotensin I radioimmunoassay. ${ }^{8)}$ In this report, the values determined by the bioassay were calculated and expressed in terms of the radioimmunoassay values. Sampling of resting peripheral vein blood PRA was done with fasting patients kept in recumbent position for at least one hour in the morning. To evaluate the reaction of renin secretion, an intravenous injection of furosemide ( $1 \mathrm{mg}$ per kilogram of body weight) followed by 2 hours' upright posture was performed. Renal vein blood was obtained through the percutaneous transfemoral catheterization. Postoperative blood pressure and PRA were measured serially using the samples of peripheral vein blood which were collected at $1,2,8$ and 24 postoperative hours, and 2, 7 and 14 postoperative days.

Rapid sequence excretory pyelography was performed as follows: the contrast medium was injected rapidly into an antecubital vein, and renal roentgenograms were taken at $1,2,3,5$ and 15 minutes. Radioisotope renography was carried out by the in jection of ${ }^{131}$ I-labeled Hippuran.9) Split renal function was studied by using the tubular rejection fraction ratio (TRFR) by Rapoport. ${ }^{103}$

Angiographic studies were done by serial abdominal aortography and selective renal angiography by the Seldinger technique. ${ }^{11)}$ The serial angiograms were obtained at a speed of 6 exposures per second during the first 2 seconds and one exposure per second during the next 10 
seconds.

\section{RESULTS}

A) Diagnosis

Fig. 1 demonstrates the percentages of the positive findings of abdominal bruit, intravenous pyelography, radioisotope renography, split renal function test and measurements of plasma renin activities at rest or after the stimulation.

(1) Abdominal bruit

The presence or absence of an abdominal bruit was examined in 70 cases. The bruit was heard in 18 cases $(46 \%)$ out of 39 with UMRAS, in $12(75 \%)$ out of 16 with BMRAS and in $4(27 \%)$ out of 15 with RBAS.

(2) Intravenous pyelography

This test was interpreted as positive when one or more of the following

Abbreviations UMRAS; unilateral renal arterial stenosis

BMRAS ; bilateral arterial stenoses RABS; renal arterial branch stenosis

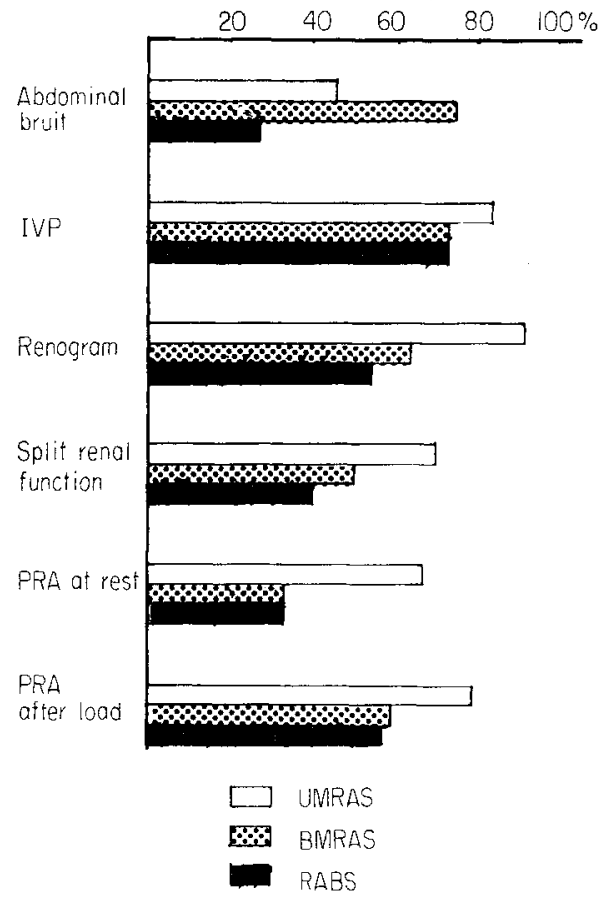

Fig. 1. The percentage of the positive findings of abdominal bruit and laboratory examinations. findings were observed: (i) A significant difference in renal contour; the longitudinal length of the right kidney was smaller than the left by $1.5 \mathrm{~cm}$ or more, or the left kidney was smaller than the right by $1.0 \mathrm{~cm}$ or more. (ii) Delayed pyelocalyceal appearance of contrast medium on the involved side in rapid sequence. (iii) Hyper-concentration of pyelogram on the involved side in delayed time. (iv) Notching of the pelvis and/or ureter suggesting collateral circulation.

Intravenous pyelography was examined in 75 cases. Abnormal findings were observed in 38 cases $(48 \%)$ ) out of 45 with UMRAS, in 11 cases $(73 \%)$ out of 15 with BMRAS and in 11 cases $(73 \%)$ out of 15 with RABS.

(3) Radioisotope renography

This test was interpreted as positive when there was a drop of initial spike and/or peak value, or delay of peak time. The radioisotope renogram was examined in 57 cases. Abnormal findings were obtained in 33 cases (92\%) out of 36 with UMRAS, in 5 cases $(63 \%)$ out of 8 with BMRAS and in 7 cases $(54 \%)$ out of 13 with RBAS.

(4) Split renal function test

This study was done in 45 cases. Urine collection from the involved kidney was impossible in 4 cases and they were removed from the following statistics. An ischemic pattern based on Rapoport TRFR was found in 18 cases $(70 \%)$ out of 26 with UMRAS and in 3 cases (40\%) out of 7 with unilateral RABS (URABS).

(5) Plasma renin activity

(a) Resting peripheral vein blood PRA

(i) Healthy persons; Resting pe. ripheral vein blood PRA in 123 healthy persons ranged from 2 to $36 \mathrm{ng} / \mathrm{ml}$ (mean and $\mathrm{SD}: 12.4 \pm 7.3 \mathrm{ng} / \mathrm{ml}$ ) as shown in Fig. 2. Ninety-three percent of the subjects (116 out of 123 persons) had the values between 5 and $30 \mathrm{ng} / \mathrm{ml}$. From these results, the normal range of resting peripheral vein blood PRA was defined 


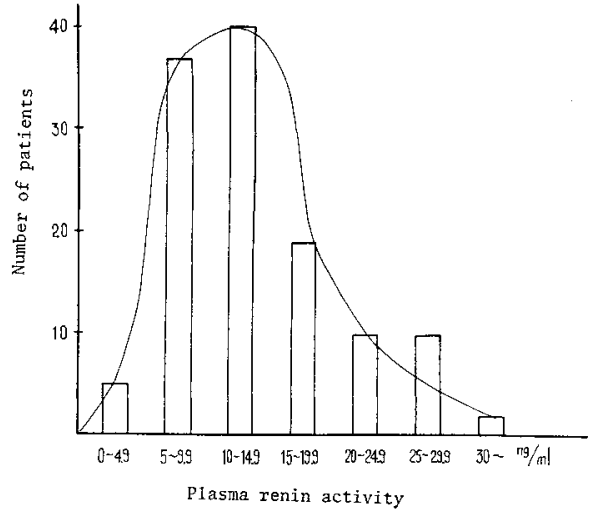

Fig. 2. Distribution of resting peripheral vein blood PRA in 123 normal subjects.

to be from 5 to $30 \mathrm{ng} / \mathrm{ml}$.

(ii) Renovascular hypertension; Resting levels of peripheral vein blood PRA were determined in 78 cases of renovascular hypertension. In two patients out of them, severe hypertensive vascular complications were observed and the antihypertensive drugs could not been discontinued. Therefore, these patients were removed from the following discussion. Seventy-six cases consisted of 45 with UMRAS, 15 with BMRAS, 14 with RABS and 2 with UMGBS. Fig. 3 demonstrates resting PRA levels in each group. The average value and $\mathrm{SD}$ were $55.2 \pm 39.8 \mathrm{ng} / \mathrm{ml}$ in UMRAS, $35.7 \pm 21.5 \mathrm{ng} /$ $\mathrm{ml}$ in BMRAS and $41.1 \pm 34.1 \mathrm{ng} / \mathrm{ml}$ in

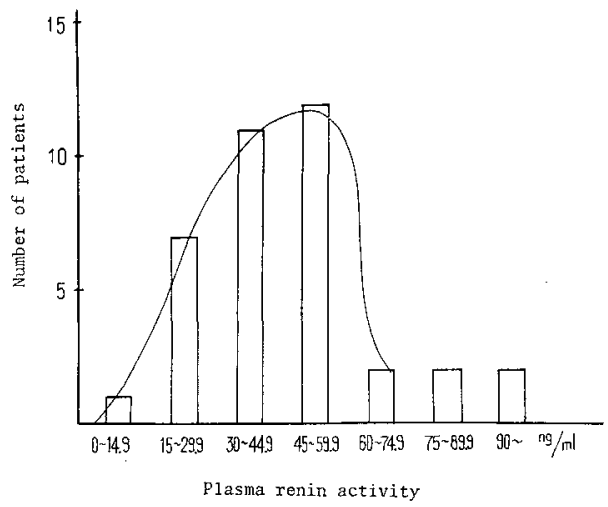

Fig. 3. Distribution of peripheral vein blood PRA after intravenous furosemide and upright posture in 37 normal subjects.
RABS. Out of 45 patients with UMRAS, $30(67 \%)$ had an elevated PRA levels. On the contrary, only 5 cases $(33 \%)$ out of 15 with BMRAS and 4 cases (28\%) out of 15 with RABS had significantly increased PRA values.

(b) Provocation test for reninsecretion

(i) Normal persons; Provocation of renin secretion was studied in 37 healthy subjects. The peripheral vein blood PRA values after the load of furosemide and upright posture ranged from 13.2 to 100 $\mathrm{ng} / \mathrm{ml}$ (average and SD; $48.9 \pm 21.9 \mathrm{ng} / \mathrm{ml}$ ). Fig. 4 demonstrates the relationship between subjects numbers and the PRA values. Thirty-one cases $(83.8 \%)$ out of 37 had the PRA values of 15 to $60 \mathrm{ng} / \mathrm{ml}$. This range of PRA was defined normal in this provocation study. ${ }^{12}$

(ii) Renovascular hypertension; The provocation test of renin secretion was carried out in 34 cases of renovascular hypertension, including 21 patients with normal and 13 with elevated resting PRA values. Fig. 5 showed the PRA levels after the stimulation in each group. Twenty-four cases $(70.6 \%)$ showed a hyperresponse of renin secretion. In evaluating the relationship between the responses

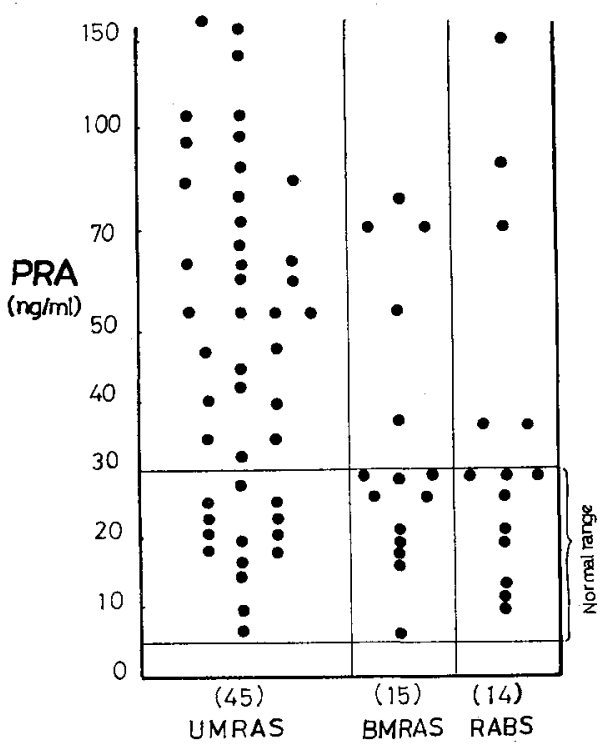

Fig. 4. Peripheral vein blood PRA levels in each type of renovascular lesions. 


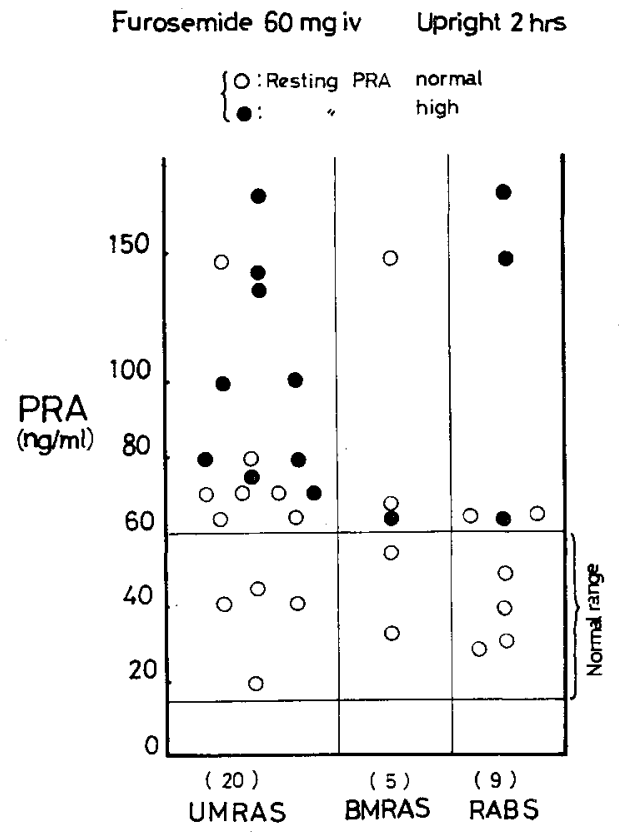

Fig. 5. Peripheral vein blood PRA levels after intravenous furosemide and upright posture in each type of renovascular hypertension.

of renin secretion and the types of renal arterial lesions, an augmented renin secretion was observed in 16 cases $(80 \%)$ out of 20 with UMRAS, in 3 cases $(60 \%)$ out of 5 with BMRAS and in 5 cases $(57 \%)$ out of 9 with RABS

The normal resting PRA levels were found in 21 patients including 11 cases with UMRAS, 4 with BMRA and 6 with RABS. The hyperresponse was found in 7 cases $(64 \%)$ out of 11 with UMRAS, in 2 cases $(50 \%)$ out of 4 with BMRAS and in 2 cases $(33 \%)$ out of 6 with RABS. The remaining $4(36 \%)$ with UMRAS, 2 $(50 \%)$ with BMRAS and $4(67 \%)$ with RA BS responded normally to the stimulus.

(c) Renal vein blood PRA

(i) Essential hypertension; Renal vein PRA was estimated in 13 patients with essential hypertension as controls. The differences between the both sides were from 0.9 to $17 \mathrm{ng} / \mathrm{ml}$ (mean 6.2), and the ratio (the value of higher side divided by that of the opposite) ranged from 1.05 to 1.45 (mean 1.23). From these results, renal vein PRA values in patients with renovascular hypertension were judged abnormal when the difference between the both sides was $17 \mathrm{ng} / \mathrm{ml}$ or higher, and/or the ratio of difference was 1.50 or greater.

(ii) Renovascularhypertension; Renal vein catheterization was performed in 40 patients with renovascular hypertension, including 23 patients with UMRAS, 7 with BMRAS, 8 with URABS, and 2 with UMCBS. In twenty-three cases with UMRAS, the difference ranged from 0 to $140 \mathrm{ng} / \mathrm{ml}$ with an average of $40.0 \mathrm{ng} / \mathrm{ml}$, and the ratio ranged from 1.0 to 3.3 with an average of 1.94. The significant difference as defined above was found in 18 patients $(78 \%)$ out of 23 with UMRAS. No lateralization was found in the remaining 5 patients (22\%). The significant lateralization was also observed in 2 cases out of 7 with BMRAS. These 2 patients revealed a severe stenotic lesion in the side with higher PRA levels by angiographic studies. In 8 patients with URABS, the difference ranged from 2.6 to $176 \mathrm{with}$ an average of $35 \mathrm{ng} / \mathrm{ml}$, and the ratio ranged from 1.1 to $3.5 \mathrm{with}$ an average of 1.75. Significant lateralization was obtained in 5 cases out of 8 with URABS. In 2 cases of UMCBS, there was a significant elevation of PRA in the side of main renal arterial stenosis.

(6) Angiography

Angiographic examinations (abdominal aortography and selective renal arteriography) were done in 79 out of 80 cases of renovascular hypertension. Angiogram revealed a unilateral renal arterial lesion in 60 cases, including 47 cases of a main renal arterial stenosis and 13 of a renal arterial branch stenosis, and bilateral renal arterial lesions in 19 cases, including 16 of bilateral main renal arterial stenosis, 2 of unilateral main renal arterial stenosis associated with contralateral renal arterial branch stenosis, and one of bilateral renal arterial branch stenosis.

Serial abdominal aortography was Jap J Med Vol 15, No l (Jan 1976) 
done in 26 cases. Renal collateral circulation was found in 19 cases (73\%), including 15 cases $(84 \%)$ out of 18 UMRAS and 4 cases $(50 \%)$ out of 8 URABS. As demonstrated in Fig. 6, peripelvic collateral was found in 14 cases $(74 \%)$ out of 19 , pericapsular collateral in 9 cases $(47 \%)$ and periureteric collateral in 4 cases $(21 \%)$.

B) Retrospective Evaluation for Surgical Gurability

(1) Result of surgical treatment

All patients had received one or more antihypertensive drugs, and 3 responded well to this treatment. Forty-nine out of 80 patients underwent surgical operation for the relief of hypertension. The renal vascular repair was attempted in 26 cases; unilateral revascularization in 21 cases and bilateral in 5 . In 5 cases with unilateral vascular repair, thrombosis developed postoperatively in the lumen of the grafted artery and their blood pressure aggravated markedly, necessitating the subsequent nephrectomy. On the other hand, nephrectomy was chosen for the first treatment in 23 cases.

Patients were judged as cured by surgical treatment when systolic blood pressure fell to $150 \mathrm{mmHg}$ or lower and diastolic blood pressure to $90 \mathrm{mmHg}$ or lower without any antihypertensive drug. The results were classified as improved,

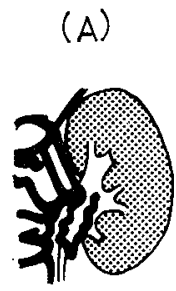

$14 / 19$
$(74 \%)$

\section{Collaterals}

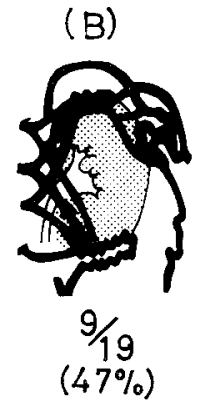

(C)
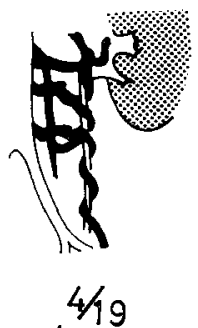

Fig. 6. Demonstration of renal collateral circulation (Abrams et al. ${ }^{13)}$ ).

(A) peripelvic collateral

(B) pericapsular collateral

(C) periureteric collateral

if the diastolic blood pressure had decreased by $20 \mathrm{mmHg}$ or more but not to the normotensive with no or only mild antihypertensive medication.

Twelve out of 21 cases with unilateral renal vascular repair were cured and 6 cases were improved postoperatively. The remaining 3 cases wer unimproved. Similar results were also obtained in unilateral nephrectomized cases. In this group, 14 out of 23 patients were cured and 8 were improved. The remaining one died. As a whole, 44 patients with unilateral renal arterial lesion underwent surgical operation, and 27 cases were cured, 13 cases improved, 3 cases unimproved and one died. On the other hand, 2 out of 5 patients with bilateral renal revascularization, in whom vascular lesion was Takayasu's aortitis, died from rupture of a pseudoaneurysm occurring on the grafted artery. In this group, only 2 patients were cured.

(2) Retrospective evalution for surgical treatment

To evaluate the prognostic value of the preoperative examinations in predicting surgical curability of renovascular hypertension, the results of operation were analysed. There were 40 patients who were cured or improved by unilateral renal vascular repair or unilateral nephrectomy. Split renal function test, measurement of PRA, and pressure gradient across the stenotic portion of renal artery are currently used to diagnose the curability of hypertension. In this study, split renal function test was done in 24 cases of 40. Ischemic findings of the affected kidney were obtained in 16 cases $(67 \%)$. In $4(50 \%)$ out of 8 cases without unilateral renal ischemic pattern, however, blood pressure returned to normal levels without any antihypertensive drug postoperatively.

Resting peripheral vein PRA was measured in 40 cases. A significantly elevated PRA was found in 27 cases (68\%). The provocation test of renin secretion by injecting furosemide $60 \mathrm{mg}$ 
iv and loading 2 hours' upright posture was carried out in 22 cases of whom 15 cases had a unilateral main renal arterial lesion and 7 unilateral renal arterial branch lesion. Hyperresponse of renin secretion was found in 12 caces $(80 \%)$ of the former and in 4 cases $(57 \%)$ of the latter. Renal vein blood PRA was measured in 24 cases. A significant elevation of renin secretion from the affected kidney was found in 75\% of them. On the contrary, no lateralization of renal vein renin was observed in unimproved patients.

It is technically difficult to estimate the pressure gradient across a stenotic portion of renal artery preoperatively. It has been described that appearance of collaterals in angiogram was an important finding to suggest the existence of pressure gradient at the stenosis. ${ }^{13)}$ In our clinic, 19 cases with collaterals underwent surgical operation, and 18 cases (95\%) were cured or improved.

From these results, it is proposed that the most reliable diagnostic and prognostic criteria for surgical curability in renovascular hypertension were the collateral circulation, elevated peripheral vein PRA with a significant lateralization of renin secretion from the affected kidney, and an ischemic pattern of split renal function test.

C) Postoperative Course of PRA and Blood Pressure

Postoperative blood pressure was followed serially in 14 nephrectomized and 10 revascularized patients, and PRA in 7 nephrectomized and 3 revascularized. All patients had been cured or improved.

(1) Postoperative course of blood pressure

Preoperative systolic blood pressure ranged from 162 to $230 \mathrm{mmHg}$ with an average of $197.9 \pm 23.6$ (SD) in nephrectomized patients and from 158 to 220 $\mathrm{mmHg}$ with an average of $190.1 \pm 16.2$ (SD) in revascularized patients. Preoperative diastolic pressure ranged from 90 to 150 $\mathrm{mmHg}$ with an average of 118.8 土16.8 (SD) in nephrectomized and from 76 to 156 $\mathrm{mmHg}$ with an average of $108.2 \pm 10.3$ (SD) in revascularized patients. There was no significant difference in the baseline levels of blood pressure between both groups.

In all patients, blood pressure returned to normal within 30 days postoperatively. There was a significant difference in the time intervals required for the normalization of postoperative blood pressure between nephrectomized and revascularized patients as shown in Fig. 7. The normalization of blood pressure following operation was more rapid in revascularized group than in nephrectomized. The mean and SD of normalization time were $18.4 \pm 6.2$ days in nephrectomized patients. On the other hand, 8 $(80 \%)$ out of 10 patients with vascular repair had normal blood pressure within 2 weeks after operation. The mean and SD of the normalization time were $9.7 \pm$ 4.4 days, being significantly shorter than that in nephrectomized patients.

(2) Postoperative course of PRA

Postoperative peripheral vein blood PRA was measured serially in 10 patients. Fig. 8 illustrated the postoperative course of PRA in 7 nephrectomized (solid circle) and in 3 revascularized patients (open circle). The PRA values were normalized in 9 patients $(90 \%)$ within the first 3 days after operation and all had normal PRAs by the 7 th day after operation. In nephrectomized patients, PRAs were rapidly normalized following operation in all patients except one in whom PRA was extremely elevated after the first

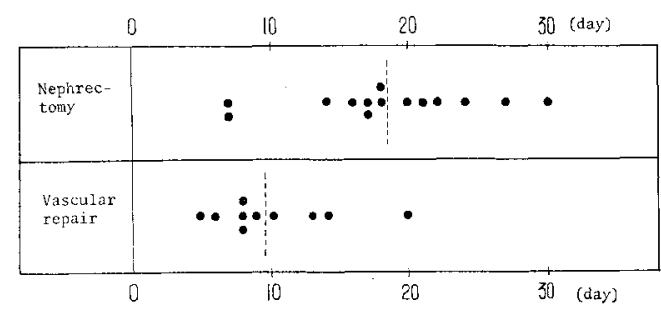

Fig. 7. Time interval for normalization of postoperative blood pressure.

Jap J Med Vol 15, No l (Jan 1976) 


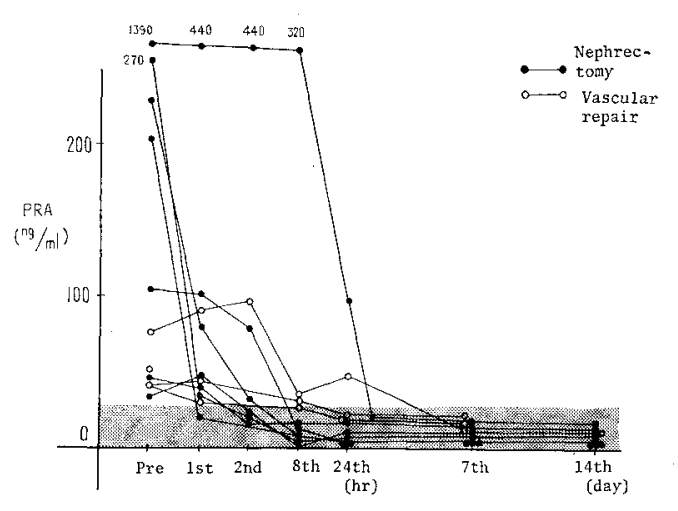

Fig. 8. Postoprative course of PRA in 10 patients with renovascular hypertension. Solid circle; nephrectomy Open circle; vascular repair

vascular reconstruction, because of thrombus formation in the grafted artery. In this case, the PRAs required 3 days to return to normal after the secondary nephrectomy. On the other hand the normalization of PRA after vascular repair was delayed and it required 24 hours in 2 patients and 7 days in the remaining one. To summarize, PRA was normalized more rapidly in nephrectomized than in revascularized patients after operation.

There was a reversed relationship between the postoperative courses of blood pressure and those of PRA in the two groups.

\section{DISCUSSION}

The incidence of renovascular hypertension is extremely difficult to determine. In 1968, Hunt $t^{1)}$ described in his review that the estimates of the incidence of this disease have ranged from 2 to $3 \%$ of the entire hypertensive population. Imai ${ }^{14}$ found in a population study in a village of northern Japan that out of 416 consecutive hypertensive patients, 13 were demonstrated angiographically to have renovascular lesion, an incidence of approximately $3 \%$.

For the selection of renovascular hypertension, the following clinical histories have been well known to be worthy of consideration ${ }^{15}$ : (1) Young hypertensive patients without a family history of hypertension, and acute or severe hypertension in patients of 55 years or older. (2) Hypertension developing after acute flank pain. (3) Sudden acceleration of chronic essential hypertension. Among our patients, 2 had a history of trauma on their body during a play of rugby game or a car-accident. They complained of their right flank pain with nausea and vomiting postraumatically, and hypertension developed with angiographically proved renal lesions.

A diagnosis of renovascular hypertension could be established by means of clinical features and the laboratory examinations including intravenous pyelography, split renal function test, estimation of PRA and angiography. It was not difficult to detect the typical renovascular hypertension due to constriction of a main renal artery by a combination of these examination procedures. On the contrary, the identification of the patient with branch arterial stenosis was very difficult. ${ }^{3}$ It has been reported by many researchers that the measurement of PRA is very useful in establishing a diagnosis of renovascular hypertension. However, only $9(36 \%)$ out of 25 cases who were encountered in our clinic for the last 3 years showed a significantly increased value of resting peripheral vein PRA. ${ }^{16}$ ) This result seems to come from the fact that half of them belonged to the patients with branch renal arterial or bilateral main renal arterial lesions in which normal PRA levels were usually obtained. Accordingly, the measurement of resting peripheral vein PRA is seldom helpful to detect the renovascular hypertension with branch arterial lesions. In the present study, the available examination procedures $(50 \%$ positive or above) in identifying this type of renovascular hypertension were intravenous pyelography, radioisotope renography and the provocation test of renin secretion by iv 
injection of furosemide followed by upright posture. However, there are a few cases without any abnormal findings in all laboratory examinations except angiography. From these results, we propose that the most reliable diagnostic procedures for the detection of the patients with renal arterial branch stenosis were angiographic studies (serial abdominal aortography and selective renal arteriography). The angiographic examination should be done in all hypertensive patients with no abnormality in PRA and other clinical findings.

Occlusive lesions have often been demonstrated in renal arteries of normotensive subjects as well as hypertensives by angiographic or postmortem examinations.5) Therefore, when a renal arterial lesion has been found, the determination of its pathophysiologic significance is very important. It is well known that split renal function studies, the determination of PRA in peripheral and renal vein, and the estimation of pressure gradient across stenotic portion of renal artery are very effective to identify potentially curable patients by surgery. However, it is very difficult to measure the pressure gradient before surgery. Accordingly, split renal function studies and the determination of PRA are used to predict the curability in many clinics.

In the present study, the excellent surgical results were obtained in the patients with increased renin secretion from the affected kidney and an ischemic pattern on the split renal function test. However, no enhancement of renin secretion was found in 20\% of cases with UMRAS and $40 \%$ of cases with URABS who were cured or improved by unilateral renal vascular repair or unilateral nephrectomy. Recently, it was also reported by us that the intravenous infusion of angiotensin II antagonist, 1-Sar-8-Ileangiotensin II, induced no depressor response in 5 cases out of 6 with renovas. cular hypertension who were cured by surgical operation. ${ }^{173}$ The present study also showed that $50 \%$ of patients without unilateral renal ischemic pattern in split renal function study was relieved by unilateral vascular repair or extirpation of the affected kidney. In the patients, decision of operation had not been made by the measurement of PRA or split renal function test. Only available diagnostic and prognostic finding for surgical curability in these cases was the demonstration of collateral circulation. The collateral circulation on angiographic study is a strong evidence of the existence of pressure gradient across the renal arterial stenosis and it is an important finding in predicting the surgical result.

There was a significant difference between postoperative courses of blood pressure and PRA in revascularized and in nephrectomized patients. The normalization of blood pressure after operation was quicker in the former than in the latter. The only difference between groups was that there remained a revascularized kidney in the former but not in the latter. This result suggests that the renal antihypertensive mechanism has been suppressed or lost in the affected kidney contributing to the hypertension and that it is restored with reestablishment of normal circulation after operation resulting in a rapid normalization of blood pressure.

\section{REFERENCES}

1) Hunt JC: Diagnosis of renovascular hypertension. Bull NY Acad Med 45 : 877, 1969.

2) Brown JJ et al: Plasma renin concentration in human hypertention. Brit Med J 2: 1215, 1965.

3) Abe K, Saito T, Otsuka Y et al: Seven cases of hypertension due to segmental renal ischemia. Jap Heart J 14: 110, 1973.

4) Simon N, Franklin SS, Bleifer KH et al: Clinical characteristics of renovascular hypertension. JAMA 220: 1209, 1972.

5) Holley KE, Hunt JG, Brown AL et al: Renal artery stenosis: A clinical-pathologic study in normotensive and hypertensive patients. Amer J Med 37 : 14. 1964.

Jap J Med Vol 15, No 1 (Jan 1976) 
6) Yoshinaga $\mathrm{K}$, Aida $\mathrm{M}$, Maebashi $\mathrm{M}$ et al: Assay of renin in peripheralblood: A modi. fication of Helmer's method for the estimation of the circulating renin. Tohoku J exp. Med. 80: 32, 1963.

7) Miura $Y$ : Improved method for measurement of renin activity in human plasma. Tohoku J exp Med 98 : 125, 1969.

8) Abe K, Otsuka Y, Saito T et al: Measure. ment of plasma renin activity by angiotensis $I$ radioimmunoassay: A modification of Haber' smethod. Jap Circulat J 36: 741, 1972 .

9) Winter CG: Unilateral renal disease and hypertension: Use of the radioactive diodrast renogram as a screening test. J Urol 78 : 107, 1957.

10) Rapoport A: Modification of the "Howard Test" for the detection of renal-artery obstruction. New Engl. J Med 263: 1195, 1960.

11) Seldinger SI: Catheter replacement of the needle in percutaneous arteriography: A new technique. Acta Radiol 39: 368, 1953.

12) Abe K, Irokawa $\mathrm{N}$, Aoyagi $\mathrm{H}$ et al: Circulating renin in essential hypertension: An evaluation of its significance in the Japanese population. Amer Heart J 89: 723, 1975.

13) Abrams HL: Pattern of collateral flow in renal ischemia. Radiology 84: 1001, 1965.

14) Imai $J$ Itoh $T$ et al: A population study of hypertension in a village of northern Japan. Jap J Rural Med 21: 182, 1972.

15) Poutasse EF et al: Arteriosclerosis and renal hypertension. JAMA 165: 1521, 1957.

16) Otsuka $Y$, Abe $K$, Saito $T$ et al: Plasma renin activity in patients with renovascular hypertension. Tohoku J exp Med 16: 149, 1975.

17) Yasujima M, Abe K, Aoyagi $H$ et al: Efects of 1-Sar-8-Ile-angiotensin II on blood pressure and plasma renin activity in various types of hypertension. Tohoku J exp Med $116: 277,1975$. 